\title{
A Hybrid Approach Integrating Multiple ICEEMDANs, WOA, and RVFL Networks for Economic and Financial Time Series Forecasting
}

\author{
Jiang Wu $\mathbb{D}$, Tengfei Zhou $(D$, and Taiyong Li $(D)$ \\ School of Economic Information Engineering, Southwestern University of Finance and Economics, Chengdu 611130, China \\ Correspondence should be addressed to Taiyong Li; litaiyong@gmail.com
}

Received 8 May 2020; Revised 11 August 2020; Accepted 6 October 2020; Published 22 October 2020

Academic Editor: Lei Xie

Copyright () 2020 Jiang Wu et al. This is an open access article distributed under the Creative Commons Attribution License, which permits unrestricted use, distribution, and reproduction in any medium, provided the original work is properly cited.

\begin{abstract}
The fluctuations of economic and financial time series are influenced by various kinds of factors and usually demonstrate strong nonstationary and high complexity. Therefore, accurately forecasting economic and financial time series is always a challenging research topic. In this study, a novel multidecomposition and self-optimizing hybrid approach integrating multiple improved complete ensemble empirical mode decompositions with adaptive noise (ICEEMDANs), whale optimization algorithm (WOA), and random vector functional link (RVFL) neural networks, namely, MICEEMDAN-WOA-RVFL, is developed to predict economic and financial time series. First, we employ ICEEMDAN with random parameters to separate the original time series into a group of comparatively simple subseries multiple times. Second, we construct RVFL networks to individually forecast each subseries. Considering the complex parameter settings of RVFL networks, we utilize WOA to search the optimal parameters for RVFL networks simultaneously. Then, we aggregate the prediction results of individual decomposed subseries as the prediction results of each decomposition, respectively, and finally integrate these prediction results of all the decompositions as the final ensemble prediction results. The proposed MICEEMDAN-WOA-RVFL remarkably outperforms the compared single and ensemble benchmark models in terms of forecasting accuracy and stability, as demonstrated by the experiments conducted using various economic and financial time series, including West Texas Intermediate (WTI) crude oil prices, US dollar/Euro foreign exchange rate (USD/EUR), US industrial production (IP), and Shanghai stock exchange composite index (SSEC).
\end{abstract}

\section{Introduction}

Economic and financial time series, such as price movements, stock market indices, and exchange rate, are usually characterized by strong nonlinearity and high complexity, since they are influenced by a number of extrinsic and intrinsic factors including economic conditions, political events, and even sudden crises [1,2]. Economic and financial time series forecasting always play a vital role in social and economic development, which is of great economic importance to both individuals and countries. Therefore, economic and financial time series forecasting is always a very active research area.

In extant research, various forecasting methods were proposed to forecast various economic and financial time series. These forecasting methods mainly include statistical and artificial intelligence (AI) approaches. The frequently used statistical approaches for economic and financial time series forecasting include the error correction model (ECM) [3], hidden Markov model (HMM) [4], random walk (RW) model [5], autoregressive moving average (ARMA) model [6], autoregressive integrated moving average (ARIMA) model [7], and generalized autoregressive conditional heteroskedasticity $(\mathrm{GARCH})$ model $[8,9]$. Lanza et al. forecasted the series of crude oil prices in two distinct areas using the ECM [3]. Hassan and Nath developed the HMM approach for forecasting stock price for interrelated markets [4]. Kilian and Taylor analyzed the advantage of RW in exchange rate forecasting [5]. Rout et al. integrated ARMA with differential evolution (DE) to develop a hybrid model for exchange rate forecasting [6]. Mondal et al. conducted a study on the effectiveness of the ARIMA model on the 
forecasting of 56 Indian stocks from different sectors [7]. Alberg et al. conducted a comprehensive analysis of the stock indices using various GARCH models, and the experimental results showed that the asymmetric GARCH model enhanced the overall prediction performance [9].

Since most economic and financial time series involve the complex characteristics of strong nonlinearity and nonstationarity, it is difficult to obtain satisfactory forecasting accuracy by statistical approaches. Hence, various AI approaches were proposed for economic and financial time series forecasting. These AI forecasting approaches include the artificial neural network (ANN) $[10,11]$, support vector machine (SVM) $[12,13]$, extreme learning machine (ELM) [14], random vector functional link (RVFL) neural network [15], and recurrent neural network (RNN) [16]. Pradhan and Kumar utilized ANN to forecast foreign exchange rate in India, and the experimental results indicated that the ANN could effectively forecast the exchange rate [10]. Das and Padhy forecasted the commodity futures contract index using the SVM, and the empirical analysis showed that the proposed model was effective and achieved the satisfactory prediction performance [12]. Li et al. made stock price prediction using the ELM, and the comparison results showed that the ELM with radial basis function (RBF) kernels achieved better prediction performance with faster speed than back propagation neural networks (BPNNs) [14]. Moudiki et al. employed quasirandomized functional link networks for various time series forecasting, and the proposed approach could generate more robust prediction results [15]. Baek and Kim employed long short-term memory (LSTM) for stock index forecasting, and the results confirmed the LSTM model had excellent prediction accuracy [16].

In order to effectively improve prediction accuracy, various hybrid forecasting models were designed for economic and financial time series forecasting. Babu and Reddy combined ARIMA and nonlinear ANN models to develop a novel hybrid ARIMA-ANN model, and the experiments on electricity price and stock index indicated that the proposed ARIMA-ANN had higher prediction accuracy [17]. Kumar and Thenmozhi compared three different hybrid models for the forecasting of stock index returns and concluded that the ARIMA-SVM model could obtain the highest prediction accuracy [18].Hsu built a hybrid model based on a back propagation neural network (BPNN) and genetic programming (GP) for stock/futures price forecasting, and the empirical analysis showed that the proposed hybrid model could effectively improve the prediction accuracy [19]. These hybrid models are able to fully take advantage of the potential of single models and, thus, obtain better prediction accuracy than single models.

Due to the complexity of original economic and financial time series, conducting forecasting on original time series is hard to obtain satisfactory prediction accuracy. To reduce the complexity of original time series, a framework of "decomposition and ensemble" is widely utilized in the field of time series forecasting. The framework includes three stages: decomposition, forecasting, and ensemble. The original time series is firstly separated into a sum of subseries, then a prediction model is used to forecast each subseries, and finally, the predictions of all the subseries are aggregated as the final prediction results. Decomposition, as the first step, is very important for enhancing the performance of the ensemble model. The widely used decomposition approaches include wavelet decomposition (WD), variational mode decomposition (VMD), and empirical mode decomposition (EMD) class methods. Lahmiri combined VMD with a general regression neural network (GRNN) to develop a novel ensemble forecasting model, and the experimental results suggested that VMD outperformed EMD for the prediction of economic and financial time series [20]. Kao et al. integrated WD, support vector regression (SVR), and multivariate adaptive regression splines (Mars) to develop an ensemble forecasting model to forecast stock price, and the proposed model obtained better prediction accuracy [21]. In the second stage of the framework of "decomposition and ensemble," various optimization approaches were introduced to enhance the performance of predictors. Li et al. proposed a ridge regression (RR) with DE to forecast crude oil prices and obtained excellent forecasting accuracy [22]. Bagheri et al. introduced quantum-behaved particle swarm optimization (QPSO) to tune the adaptive network-based fuzzy inference system (ANFIS) for financial time series forecasting [23]. Wang et al. employed brain storm optimization (BSO) algorithm to optimize SVR, and the results indicated that the developed approach was effective in stock market analysis [24].

In the "decomposition and ensemble" framework, decomposition approaches and prediction approaches influence the final prediction results greatly. Considering the powerful decomposition ability of ICEEMDAN, the excellent search efficiency of WOA, and the accurate forecasting ability of the RVFL network, we develop a novel ensemble prediction model integrating multiple ICEEMDANs, WOA, and the RVFL network, namely, MICEEMDAN-WOARVFL, for economic and financial time series forecasting. Firstly, ICEEMDAN with random parameters is utilized to divide the original economic and financial time series into a sum of subseries. Secondly, the RVFL network is applied to forecast each decomposed subseries individually, and WOA is used to optimize the parameter values of the RVFL network simultaneously. Finally, the predictions of all individual subseries are aggregated as the prediction values of one process of decomposition and ensemble. From our observations, we find that the decomposition in the first stage has some disadvantages of the uncertainties with a quite randomness, which can lead to the difference and instability of the prediction results. In addition, extensive literature has shown that combining multiple forecasts can effectively enhance prediction accuracy $[25,26]$. Therefore, we randomize the decomposition parameter values of ICEEMDAN in the first stage, repeat the abovementioned processes multiple times, and integrate the results of multiple decompositions and ensembles as the final prediction values. We expect that the multiple decomposition strategy can reduce the randomness of one single decomposition and further improve the ensemble prediction stability and accuracy. 
The main contributions of this paper are as follows: (1) we propose a new multidecomposition and self-optimizing ensemble prediction model integrating multiple ICEEMDANs, WOA, and RVFL networks for economic and financial time series forecasting. As far as we know, this is the first time that the novel combination is developed for economic and financial time series forecasting. (2) To further enhance forecasting accuracy and stability, we utilize multiple differentiated ICEEMDANs to decompose original economic and financial time series and, finally, ensemble the predictions of all decompositions as the final predictions. (3) WOA is firstly introduced to optimize various parameters of RVFL networks. (4) The empirical results on four different types of economic and financial time series show that our proposed MICEEMDAN-WOA-RVFL significantly enhances the prediction performance in terms of forecasting accuracy and stability.

The novelty of the proposed MICEEMDAN-WOARVFL is three-fold: (1) a novel hybrid model integrating multiple ICEEMDANs, WOA, and RVFL networks is designed for economic and financial time series forecasting; (2) the multiple decomposition strategy is firstly proposed to overcome the randomness of one single decomposition and to improve prediction accuracy and stability; and (3) WOA is first applied to optimizing RVFL networks to improve the performance of individual forecasting.

The remainder of the paper is organized as follows. Section 2 offers a brief introduction to the ICEEMDAN, WOA, and RVFL network. Section 3 provides the architecture and the detailed implementation of the proposed MICEEMDAN-WOA-RVFL. Section 4 analyzes the empirical results on various economic and financial time series forecasting. Section 5 discusses some details of the developed prediction model, and Section 6 concludes this paper.

\section{Preliminaries}

2.1. Improved Complete Ensemble Empirical Mode Decomposition with Adaptive Noise (ICEEMDAN). Empirical mode decomposition (EMD), an adaptive time-frequency analysis approach for nonstationary signals, was designed by Huang et al. [27]. EMD separates original time series into a sum of "intrinsic mode functions" (IMFs) and one residue, and thus, it can simplify time series analysis. Due to some drawbacks of EMD, such as mode mixing, EEMD [28] and CEEMDAN [29] have been proposed to improve decomposition performance and applied in various fields [30-32]. In spite of that, these decomposition methods still have some new problems. To solve these problems, an improved CEEMDAN (ICEEMDAN) was developed by Colominas et al. [33].

Let $E_{k}(\cdot)$ be the operator which generates the $k$ th mode using EMD, $M(\cdot)$ be the operator which generates the local mean of the series, and $w_{(i)}$ be a realization of zero mean unit variance noise. When $x$ is the original signal, the detailed decomposition process of ICEEMDAN is as follows: (i) Step 1: employ EMD to compute the local means of $I$ realizations $x^{(i)}=x+\beta_{0} E_{1}\left(w_{(i)}\right)$ to achieve the first residue $r_{1}=M\left(x^{(i)}\right)$, and $\beta_{0}>0$

(ii) Step 2: calculate the first $I M F_{1}=x-r_{1}$

(iii) Step 3: calculate the average of local means of the realizations as the second residue: $r_{2}=\left(r_{1}+\beta_{1} E_{2}\left(w^{(i)}\right)\right)$

(iv) Step 4: compute the $k$ th residue for $k=3, \ldots, K$ : $r_{k}=M\left(r_{k-1}+\beta_{k-1} E_{k}\left(w^{(i)}\right)\right)$

(v) Step 5: go to step 4 for next $k$

Since ICEEMDAN can effectively decompose the original time series, it has been frequently introduced into various time series forecasting [34-36]. In our study, we employ ICEEMDAN to separate the original economic and financial time series into a sum of simpler subseries for subsequent forecasting.

2.2. Whale Optimization Algorithm (WOA). Whale optimization algorithm (WOA) is a type of optimization method and outperforms particle swarm optimization (PSO), ant colony optimization (ACO), gravitational search algorithm (GSA), and fast evolutionary programming (FEP) in the optimization performance $[37,38]$. Simulating the hunting process of whales, WOA includes three main operators: encircling prey, bubble-net foraging, and search for prey. In each iteration, individuals update their positions toward the best individual in the last iteration, which can be formulated as follows:

$$
P(t+1)=P_{\text {best }}(t)-A \cdot\left|C \cdot P_{\text {best }}(t)-P(t)\right|,
$$

where $t$ represents the th iteration, $A$ and $C$ are two coefficient vectors, $P_{\text {best }}$ is the best individual so far, $P$ is the position of an individual, $\|$ represents the absolute value, and - indicates an element-by-element multiplication.

In the exploitation (bubble-net foraging) phase, the individual position is updated based on its distance to the best individual by simulating the helix-shaped movement of whales, which is formulated as follows:

$$
P(t+1)=D \cdot e^{b l} \cdot \cos (2 \pi l)+P_{\text {best }}(t),
$$

where $D=\left|P_{\text {best }}(t)-P(t)\right|$ represents the distance between the best individual obtained so far and the $i$ th individual, $l$ is a random number in $[-1,1]$, and $b$ is a constant which is used to define the shape of logarithmic spiral.

In exploration (search for prey) phase, the individual position is updated using a randomly selected individual. The mathematical model is follows:

$$
P(t+1)=P_{\text {rand }}-A \cdot\left|C \cdot P_{\text {rand }}-P(t)\right|,
$$

where $P_{\text {rand }}$ represents a randomly selected individual.

The detailed flowchart of WOA is illustrated in Figure 1, where $p$ is a random number in $[0,1]$. Due to its very competitive search ability, WOA has been widely applied in various fields [39-41]. Therefore, we consider taking advantage of the effective search ability of WOA to seek the optimal parameters for RVFL networks. 


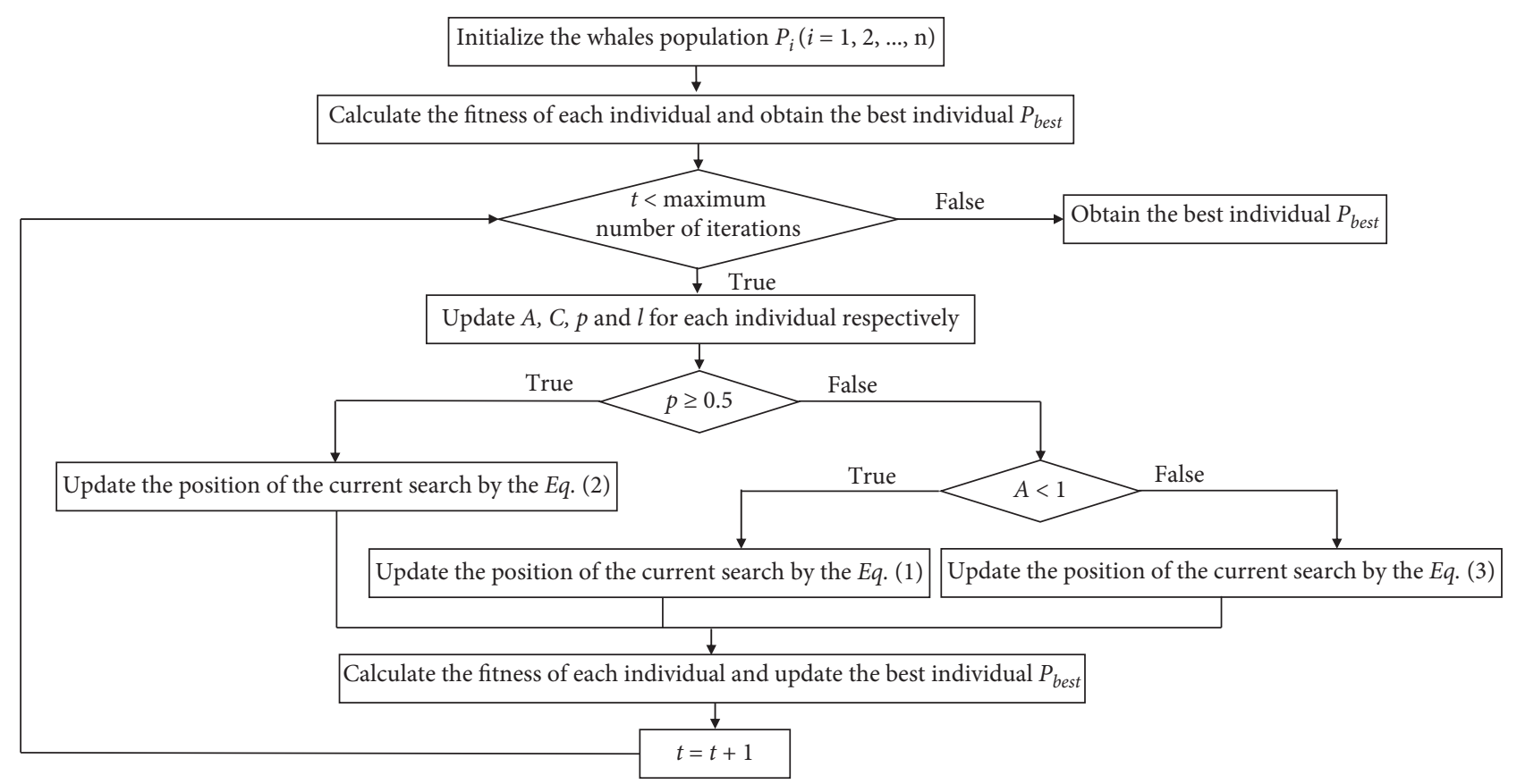

FIgURE 1: The flowchart of whale optimization algorithm (WOA).

2.3. Random Vector Functional Link (RVFL) Neural Network. As a kind of modification of the multilayer perceptron (MLP) model, the random vector functional link (RVFL) neural network was proposed by Pao et al. [42]. The RVFL neural network is able to overcome the slow convergence, overfitting, and local minimum inherently in the traditional gradient-based learning algorithms. Like MLP, the architecture of the RVFL neural network includes three layers, which is illustrated in Figure 2. The main modification in the RVFL network lies in the connection in the network structure. Since the RVFL network has the direct connections from the input layer to the output layer, it can perform better compared to no direct link $[43,44]$.

The neurons in the hidden layer, known as enhancement nodes, calculate the sum of all the output of the input layer neurons and obtain their output with an activation function:

$$
h_{m}=g\left(\sum_{n=1}^{N} w_{n m} i_{n}+b_{m}\right)
$$

where $w_{n m}$ denotes the weight between $i_{n}$ and $h_{m}, b_{m}$ represents the bias of the $m$ th neuron in hidden layer, and $g(\cdot)$ represents an activation function.

The output layer neurons integrate all the output from the hidden layer and input layer neurons, and the final output is

$$
o_{l}=\sum_{m=1}^{M} w_{m l} h_{m}+\sum_{n=1}^{N} w_{n l} i_{n},
$$

where $w_{m l}$ represents the weight between $h_{m}$ and $o_{l}$ and $w_{n l}$ indicates the weight between $i_{n}$ and $o_{l}$.

To enhance the training efficiency, the RVFL neural network utilizes a given distribution to fix the values of $w_{n m}$ and $b_{m}$ and obtain the weights of $w_{m l}$ and $w_{n l}$ by minimizing the system error:

$$
E=\frac{1}{2 P} \sum_{j=1}^{P}\left(t^{(j)}-B d^{(j)}\right)^{2},
$$

where $P$ indicates the number of training samples and $t$ are the target values, $B$ is the combination of $w_{m l}$ and $w_{n l}$, and $d$ represents a combined vector.

The RVFL neural network has demonstrated an extremely efficient and fast forecasting ability and has been frequently used in time series forecasting [44, 45].

\section{MICEEMDAN-WOA-RVFL: The Proposed Approach for Economic and Financial Time Series Forecasting}

Referring to the framework of "decomposition and ensemble," we design a multidecomposition and self-optimizing hybrid model that integrates multiple ICEEMDANs, WOA, and RVFL networks, termed as MICEEMDAN-WOA-RVFL, to forecast economic and financial time series. The architecture of the proposed hybrid model is illustrated in Figure 3.

Our proposed MICEEMDAN-WOA-RVFL takes advantage of the idea of "divide and conquer" that was frequently used in time series forecasting, image processing, fault diagnosis, and so on [46-53]. A procedure of "decomposition and ensemble" in the MICEEMDAN-WOARVFL is as follows:

(i) Stage 1: decomposition: ICEEMDAN is employed to separate original time series into several subseries (i.e., several IMFs and one residue). 


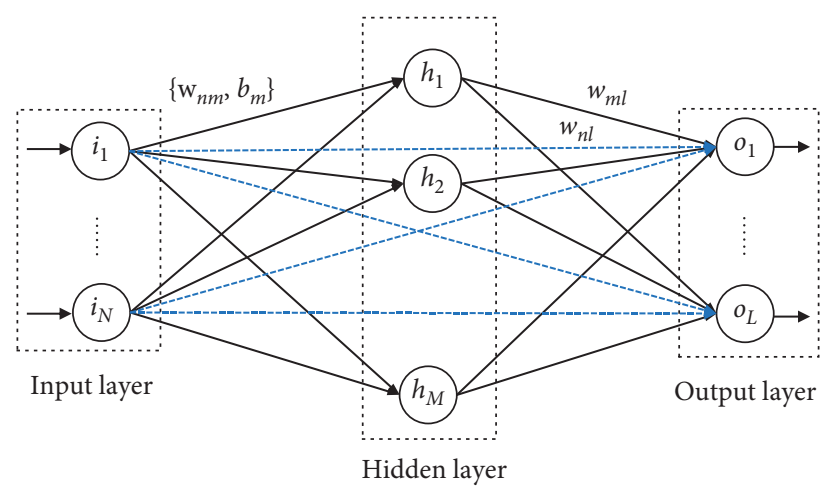

FIgURE 2: The architecture of the random vector functional link (RVFL) neural network.

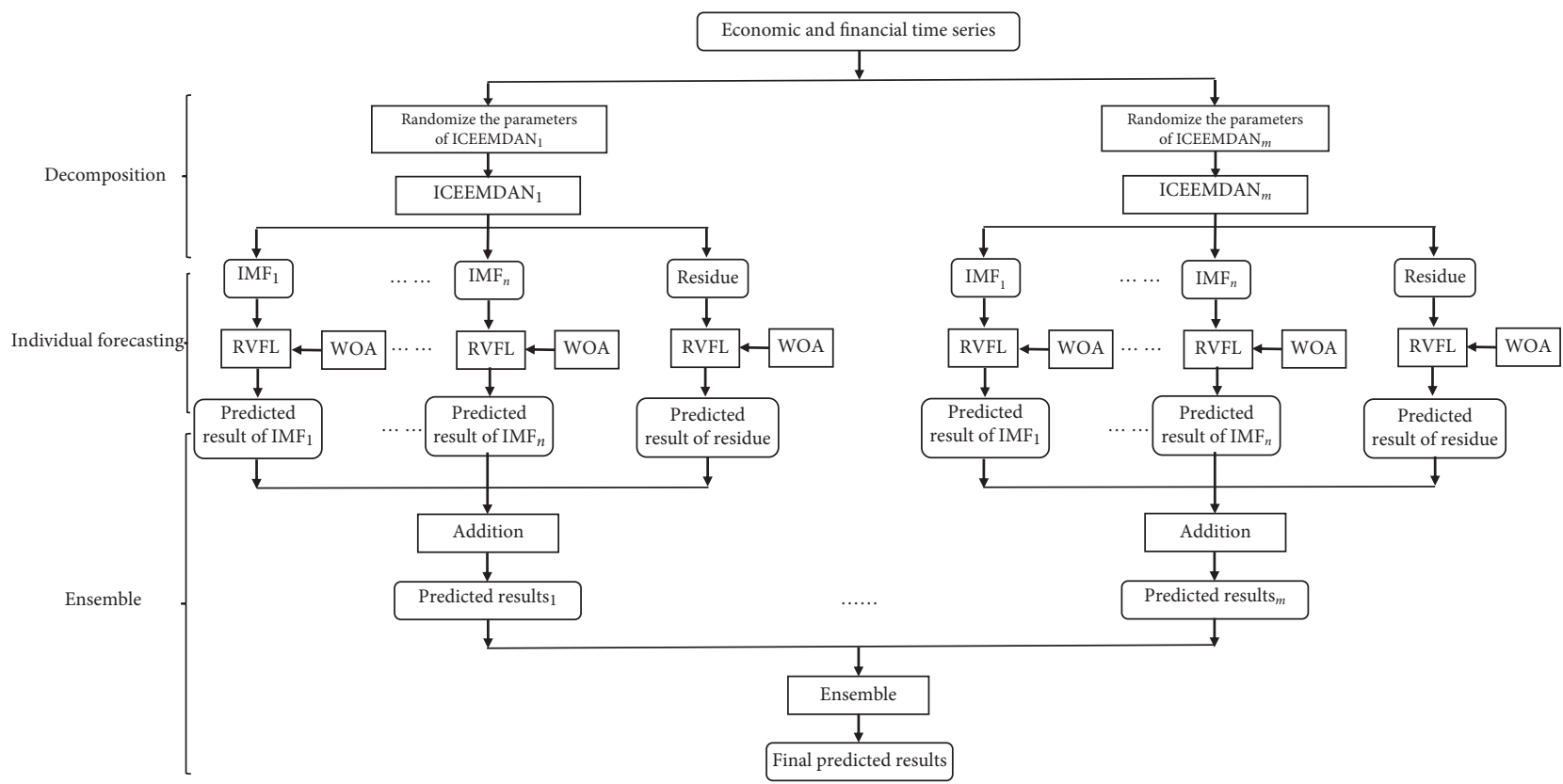

Figure 3: The flowchart of the proposed MICEEMDAN-WOA-RVFL.

(ii) Stage 2: individual forecasting. Each decomposed subseries is divided into a training dataset and a testing dataset, then the RVFL network with WOA optimization is developed on each training dataset independently, and finally, the developed RVFL model is used to each testing dataset. The reason why we select the RVFL network as the predictor is its powerful forecasting ability in extant research $[15,34,44]$. Since the parameter setting of the RVFL network plays an important role in the prediction performance, we introduce WOA to seek the optimal parameter values for the RVFL network in the forecasting stage.

(iii) Stage 3: ensemble: the predictions of all the decomposed subseries are aggregated as the final prediction results of one "decomposition and ensemble" using addition aggregation.
In this study, to enhance both accuracy and stability of final prediction, we generate random values for the decomposition parameters of ICEEMDAN in the decomposition stage, including number of realizations $(\mathrm{Nr})$, noise standard deviation $(N s d)$, and maximum number of sifting iterations $\left(\operatorname{Max}_{s i}\right)$, for each decomposition, and repeat the procedure of "decomposition and ensemble" $M$ times and, finally, combine all the results of multiple "decompositions and ensembles" using the RMSE-weighted method as the final prediction results. The corresponding weight of $i$ th forecasting model is as follows:

$$
\text { Weight }_{i}=\frac{1 / \text { RMSE }_{i}}{\sum_{j=1}^{M} 1 / \text { RMSE }_{j}},
$$

where $M$ denotes the number of individual models and $R M S E_{i}$ indicates the RMSE value of the $i$ th forecasting model in the training process. 
Although some recent studies also employ the RVFL network for time series forecasting, they obviously differ from the current study in the decomposition technique and network optimization: (1) they divide the original time series using WD or EMD; (2) they construct RVFL networks using the fixed parameter values. Unlike the previous studies, our study decomposes economic and financial time series using ICEEMDAN and searches the optimal parameter values of RVFL networks based on WOA. Furthermore, the previous research mainly focuses on dividing original time series by one single decomposition or dual decomposition $[20,35,54]$. In dual decomposition, the original signal is first decomposed into several components, and then, the high-frequency components continue to be decomposed into other components using the same or different decomposition method. Essentially, the dual decomposition process belongs to one decomposition, just including two different decomposition stages. Unlike the previous research, one main improvement in this study is the multiple decomposition strategy, which can successfully overcome the randomness of one single decomposition and further improve the prediction accuracy and stability of the developed forecasting approach. To our knowledge, it is the first time that the multiple decomposition strategy is developed for the forecasting of economic and financial time series.

\section{Experimental Results}

4.1. Data Description. As we know, economic and financial time series are influenced by various factors, sometimes raising and dropping down in a short time. The dramatic fluctuations usually lead to the significant nonlinearity and nonstationarity of the time series. To comprehensively evaluate the effectiveness of the proposed MICEEMDAN-WOA-RVFL, we choose four different time series, including the West Texas Intermediate crude oil spot price (WTI), US dollar/Euro foreign exchange rate (USD/EUR), US industrial production (IP), and Shanghai stock exchange composite index (SSEC), as the experimental datasets. The first three datasets can be accessed via the website of St. Louis Fed Research [55], and the last one can be obtained via the website of NetEase [56].

Each time series is separated into two subdatasets: the first $80 \%$ for training and the last $20 \%$ for testing. Table 1 shows the divided samples of the abovementioned four economic and financial time series.

We utilize ICEEMDAN to decompose these time series into groups of relatively simple subseries. Figure 4 offers an example of the decomposition of the WTI dataset using ICEEMDAN.

4.2. Evaluation Indices. In this study, we use four evaluation metrics, including the mean absolute percent error (MAPE), the root mean squared error (RMSE), the directional statistic (Dstat), and the Diebold-Mariano (DM) test, to assess the performance of the proposed model. Among them, MAPE and RMSE are used to assess the forecasting error, defined as follows:

$$
\begin{aligned}
& \text { MAPE }=\sum_{t=1}^{N}\left|\frac{O_{t}-P_{t}}{O_{t}}\right| \times \frac{100}{N}, \\
& \text { RMSE }=\sqrt{\frac{1}{N} \sum_{t=1}^{N}\left(O_{t}-P_{t}\right)^{2},}
\end{aligned}
$$

where $N$ is the size of the evaluated samples, $O_{t}$ denotes the actual values, and $P_{t}$ represents the predicted values at time $t$. The lower the values of RMSE and MAPE, the better the prediction models.

The Dstat indicates the performance of direction prediction, which is formulated as follows:

$$
D_{\text {stat }}=\frac{1}{N} \sum_{i=1}^{N} d_{i} \times 100 \%,
$$

where $d_{i}=1$ if $\left(P_{t+1}-O_{t}\right)\left(O_{t+1}-O_{t}\right) \geq 0$; otherwise, $d_{i}=0$. A higher value of $D_{\text {stat }}$ indicates a more accurate direction prediction.

Furthermore, to test the significance of the prediction performance of pairs of models, we employ the Diebold-Mariano (DM) test in this study.

4.3. Experimental Settings. In this study, we compare the proposed MICEEMDAN-WOA-RVFL with several state-ofthe-art forecasting models, including the single models and the ensemble models. Among all these models, the single models include one popular statistical model, RW, and two popular AI models. BPNN and least square SVR (LSSVR). The ensemble models derive from the combination of the single models and the decomposition method ICEEMDAN.

The detailed parameters of all prediction models, decomposition approach ICEEMDAN, and optimization method WOA in the experiments are shown in Table 2. The parameter values of BPNN, LSSVR, RVFL, and ICEEMDAN refer to the previous literature $[22,34,45]$.

All experiments were conducted using Matlab R2019b on a PC with 64 bit Microsoft Windows 10, 8 GB RAM, and $1.8 \mathrm{GHz}$ i7-8565U CPU.

4.4. Results and Analysis. We compare the forecasting performance of six prediction models, including three single models.

(RW, LSSVR, and BPNN) and three ensemble modes (ICEEMDAN-RW, ICEEMDAN-LSSVR, and ICEEMDANBPNN) with that of our proposed MICEEMDAN-WOARVFL in terms of MAPE, RMSE, and Dstat. Due to the different horizons, we train different forecasting models separately. That is to say, we use the proposed scheme for different horizons to train different models. Tables 3-5 report the experimental results in terms of each evaluation index with 1-, 3-, and 6-horizon, respectively.

From Table 3, we can see that the proposed MICEEMDAN-WOA-RVFL obtains the lowest (the best) MAPE values with all the horizons in all the datasets. RW obtains the best MAPE values with all the horizons in all the datasets among all the compared single models, 
TABLe 1: Samples of economic and financial time series.

\begin{tabular}{|c|c|c|c|c|}
\hline Dataset & Type & Dataset & Size & Date \\
\hline WTI & Daily & $\begin{array}{l}\text { Sample set } \\
\text { Training set } \\
\text { Testing set }\end{array}$ & $\begin{array}{l}8641 \\
6912 \\
1729\end{array}$ & $\begin{array}{l}\text { 2 January 1986 15 April } 2020 \\
\text { 2 January 1986 24 May } 2013 \\
28 \text { May 2013 15 April } 2020\end{array}$ \\
\hline USD/EUR & Daily & $\begin{array}{l}\text { Sample set } \\
\text { Training set } \\
\text { Testing set }\end{array}$ & $\begin{array}{l}5341 \\
4272 \\
1069 \\
\end{array}$ & $\begin{array}{l}\text { 4 January 1999 10 April } 2020 \\
\text { 4 January 1999 30 December } 2015 \\
\text { 31 December 2015 10 April } 2020\end{array}$ \\
\hline IP & Monthly & $\begin{array}{l}\text { Sample set } \\
\text { Training set } \\
\text { Testing set }\end{array}$ & $\begin{array}{l}1215 \\
972 \\
243\end{array}$ & $\begin{array}{c}\text { January 1919 Match } 2020 \\
\text { January 1919 December } 1999 \\
\text { January 2000 Match } 2020\end{array}$ \\
\hline SSEC & Daily & $\begin{array}{l}\text { Sample set } \\
\text { Training set } \\
\text { Testing set }\end{array}$ & $\begin{array}{l}7172 \\
5737 \\
1435\end{array}$ & $\begin{array}{c}19 \text { December 1990 21 April } 2020 \\
19 \text { December 1990 4 June } 2014 \\
5 \text { June 2014 21 April } 2020\end{array}$ \\
\hline
\end{tabular}
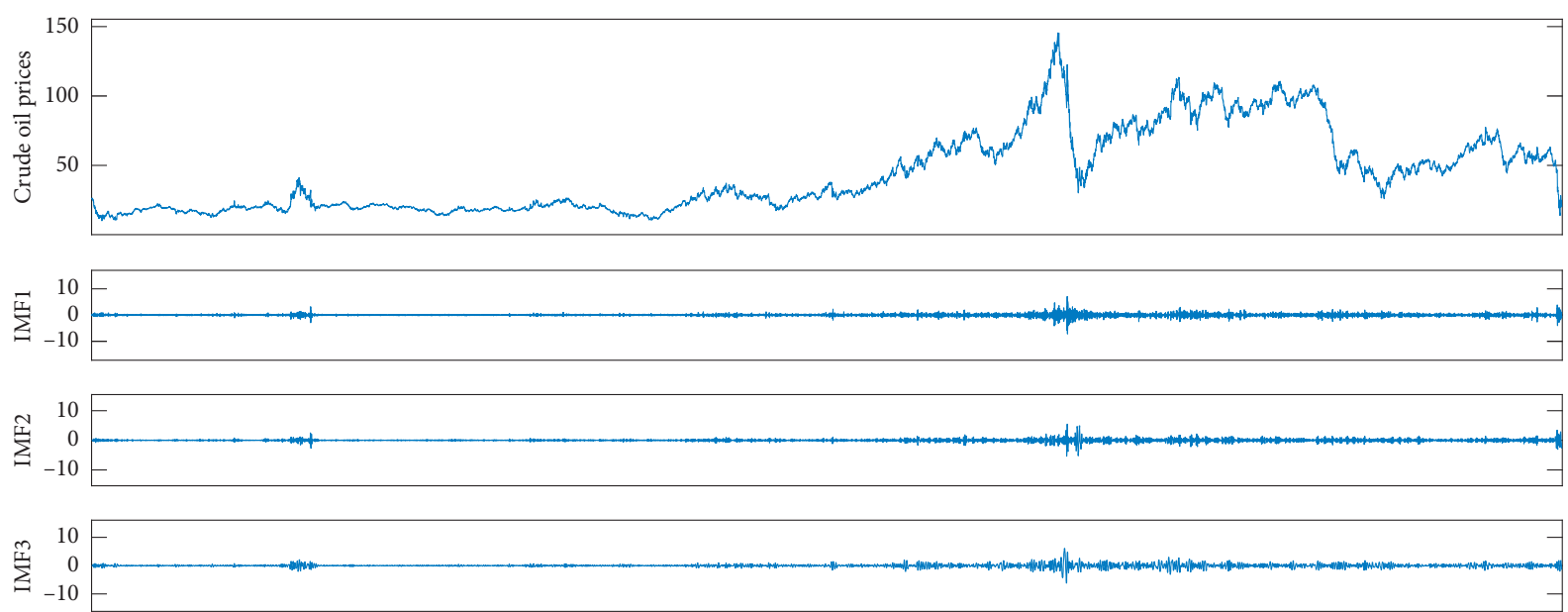

$\sum_{-10}^{+10} \begin{array}{r}10 \\ -5\end{array}$

点 $\begin{array}{r}10 \\ 0 \\ -10\end{array}$

$\sum_{i} \begin{array}{r}20 \\ 0 \\ -20\end{array}$

至 $\begin{array}{r}20 \\ 0 \\ -20\end{array}=$

$\sum_{-20}^{\infty} \begin{array}{r}20 \\ 0\end{array}-$

退 $\begin{array}{r}20 \\ 0 \\ -20\end{array}$

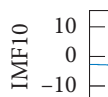

屆 $\begin{array}{r}20 \\ 0 \\ -20\end{array}-$

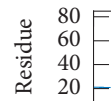

Jan 02, 1986 Apr 25, 1990 Aug 19, 1994

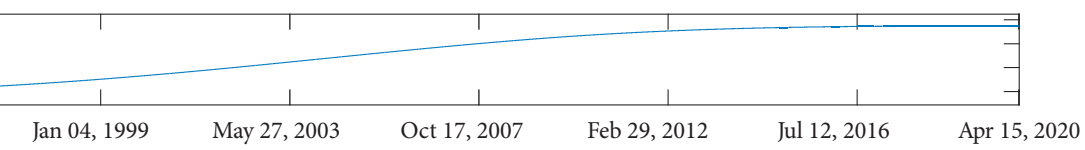

FIgURE 4: The WTI crude oil price series and the corresponding decomposed subseries by ICEEMDAN. 
TABLE 2: The settings for the parameters.

\begin{tabular}{|c|c|c|}
\hline Method & Parameters & Description \\
\hline ICEEMDAN & $\begin{array}{c}N s d=0.2 \\
N r=100 \\
\operatorname{Max}_{s i}=5000\end{array}$ & $\begin{array}{c}\text { Noise standard deviation } \\
\text { Number of realizations } \\
\text { Maximum number of sifting iterations }\end{array}$ \\
\hline LSSVR & $\begin{array}{c}R p=2^{\{-10,-9, \ldots, 11,12\}} \\
W i d_{R B F}=2^{\{-10,-9, \ldots, 11,12\}}\end{array}$ & $\begin{array}{l}\text { Regularization parameter } \\
\text { Width of the RBF kernel }\end{array}$ \\
\hline BPNN & $\begin{array}{c}N_{h e}=10 \\
M a x_{t e}=1000 \\
L r=0.0001\end{array}$ & $\begin{array}{c}\text { Number of hidden neurons } \\
\text { Maximum training epochs } \\
\text { Learning rate }\end{array}$ \\
\hline WOA & $\begin{array}{c}P o p=40 \\
\operatorname{Max}_{\text {gen }}=100\end{array}$ & $\begin{array}{c}\text { Population size } \\
\text { Maximum generation }\end{array}$ \\
\hline $\begin{array}{l}\text { MICEEMDAN-WOA- } \\
\text { RVFL }\end{array}$ & $\begin{array}{c}N s d=[0.01,0.4] \\
N r=[50,500] \\
\operatorname{Max}_{s i}=[2000,8000] \\
N_{h e}=[5,30] \\
\text { Func }=\{\text { sigmoid, sine, hardlim, tribas, radbas, sign }\} \\
\text { Mod }=1: \text { Regularized least square, } \\
\text { 2: Moore-Penrose pseudoinverse } \\
\text { Lag }=[3,20] \\
\text { Bias }=\{\text { true, false }\} \\
\text { Rand }=\{1: \text { Gaussian, } 2: \text { Uniform }\} \\
\text { Scale }=[0.1,1] \\
\text { ScaleMode }=\{1: \text { Scale the features for all neurons, } \\
\text { 2: Scale the features for each hidden neuron, } \\
\text { 3: Scale the range of the randomization for uniform } \\
\text { diatribution }\}\end{array}$ & $\begin{array}{c}\text { Noise standard deviation in ICEEMDAN } \\
\text { Number of realizations in ICEEMDAN } \\
\text { Maximum number of sifting iterations in } \\
\text { ICEEMDAN } \\
\text { Number of hidden neurons in RVFL } \\
\text { Activation function in RVFL } \\
\text { Mode in RVFL } \\
\text { Lag in RVFL } \\
\text { Bias in RVFL } \\
\text { Random type in RVFL } \\
\text { Scale value in RVFL } \\
\text { Scale mode in RVFL }\end{array}$ \\
\hline
\end{tabular}

TABLE 3: The mean absolute percent error (MAPE) values of different prediction models.

\begin{tabular}{|c|c|c|c|c|c|c|c|c|}
\hline Dataset & Horizon & MICEEMDAN -WOA-RVFL & RW & LSSVR & BPNN & ICEEMDAN -RW & ICEEMDAN -LSSVR & ICEEMDAN -BPNN \\
\hline \multirow{3}{*}{ WTI } & 1 & 0.0036 & 0.0176 & 0.0177 & 0.0182 & 0.0200 & 0.0044 & 0.0055 \\
\hline & 3 & 0.0080 & 0.0307 & 0.0309 & 0.0320 & 0.0331 & 0.0092 & 0.0094 \\
\hline & 6 & 0.0113 & 0.0441 & 0.0449 & 0.0457 & 0.0458 & 0.1202 & 0.0130 \\
\hline \multirow{3}{*}{ USD/EUR } & 1 & 0.0006 & 0.0034 & 0.0034 & 0.0034 & 0.0044 & 0.0010 & 0.0010 \\
\hline & 3 & 0.0015 & 0.0063 & 0.0063 & 0.0063 & 0.0071 & 0.0020 & 0.0021 \\
\hline & 6 & 0.0022 & 0.0087 & 0.0088 & 0.0087 & 0.0094 & 0.0032 & 0.0031 \\
\hline \multirow{3}{*}{ IP } & 1 & 0.0012 & 0.0049 & 0.0057 & 0.0056 & 0.0053 & 0.0024 & 0.0022 \\
\hline & 3 & 0.0023 & 0.0102 & 0.0140 & 0.0123 & 0.0104 & 0.0033 & 0.0033 \\
\hline & 6 & 0.0032 & 0.0183 & 0.0279 & 0.0235 & 0.0184 & 0.0043 & 0.0049 \\
\hline \multirow{3}{*}{ SSEC } & 1 & 0.0020 & 0.0096 & 0.0096 & 0.0097 & 0.0111 & 0.0026 & 0.0026 \\
\hline & 3 & 0.0044 & 0.0178 & 0.0178 & 0.0179 & 0.0185 & 0.0047 & 0.0051 \\
\hline & 6 & 0.0065 & 0.0259 & 0.0259 & 0.0269 & 0.0268 & 0.0072 & 0.0074 \\
\hline
\end{tabular}

demonstrating it is better than LSSVR and BPNN for the forecasting of economic and financial time series. Of all the ensemble models, the ICEEMDAN-LSSVR model and ICEEMDAN-BPNN model obtain the close MAPE values, obviously better than the ICEEMDAN-RW.

The RMSE values of the four time series datasets are listed in Table 4. From this table, we can find that the proposed MICEEMDAN-WOA-RVFL outperforms all the single and ensemble models with all the horizons in all the datasets. The statistical model RW obtains the best RMSE values in 10 out of 12 cases, demonstrating that it is more suitable for economic and financial time series forecasting than LSSVR and BPNN among all the single models. As to the ensemble models, the proposed MICEEMDAN-WOA-RVFL obtains the lower RMSE values than the compared ensemble models, demonstrating that the former is more effective for economic and financial time series forecasting.

Table 5 shows the directional statistics $D_{\text {stat }}$, and we can see that the MICEEMDAN-WOA-RVFL achieves the highest $D_{\text {stat }}$ values in all the 12 cases, indicating that it has better performance of direction forecasting. Amongst the single prediction models, LSSVR and RW obtain the best $D_{\text {stat }}$ values in 5 cases, respectively, better than the BPNN. Similarly, the ICEEMDAN-LSSVR model and ICEEMDANBPNN model obtain the close $D_{\text {stat }}$ values, obviously better than the ICEEMDAN-RW model in all the 12 cases.

From the all prediction results, we can find that all the ensemble prediction models except ICEEMDAN-RW greatly outperform the corresponding single prediction models in all the 12 cases, showing that the framework of decomposition and ensemble is an effective tool for improving the forecasting 
TABLE 4: The root mean squared error (RMSE) values of different prediction models.

\begin{tabular}{|c|c|c|c|c|c|c|c|c|}
\hline Dataset & Horizon & MICEEMDAN -WOA-RVFL & RW & LSSVR & BPNN & ICEEMDAN -RW & ICEEMDAN -LSSVR & ICEEMDAN -BPNN \\
\hline \multirow{3}{*}{ WTI } & 1 & 0.2715 & 1.3196 & 1.3228 & 1.3744 & 1.8134 & 0.3467 & 0.4078 \\
\hline & 3 & 0.5953 & 2.1784 & 2.1929 & 2.2867 & 2.5041 & 0.6754 & 0.7620 \\
\hline & 6 & 0.8146 & 3.0737 & 3.1271 & 3.1845 & 3.2348 & 0.8692 & 0.9462 \\
\hline \multirow{3}{*}{ USD/EUR } & 1 & 0.0009 & 0.0051 & 0.0052 & 0.0052 & 0.0099 & 0.0015 & 0.0016 \\
\hline & 3 & 0.0022 & 0.0091 & 0.0092 & 0.0092 & 0.0129 & 0.0031 & 0.0031 \\
\hline & 6 & 0.0033 & 0.0127 & 0.0128 & 0.0127 & 0.0154 & 0.0047 & 0.0046 \\
\hline \multirow{3}{*}{ IP } & 1 & 0.2114 & 0.7528 & 0.8441 & 0.8230 & 0.8205 & 0.3861 & 0.4175 \\
\hline & 3 & 0.3875 & 1.4059 & 1.8553 & 1.8311 & 1.4401 & 0.5533 & 0.5294 \\
\hline & 6 & 0.5340 & 2.4459 & 3.6015 & 2.8116 & 2.4569 & 0.6408 & 0.8264 \\
\hline \multirow{3}{*}{ SSEC } & 1 & 10.8115 & 50.0742 & 49.8800 & 50.8431 & 63.3951 & 12.6460 & 13.7928 \\
\hline & 3 & 22.2983 & 89.1834 & 88.5290 & 89.1781 & 93.2887 & 25.2526 & 29.7784 \\
\hline & 6 & 35.7201 & 131.6386 & 131.3487 & 133.5647 & 137.2598 & 40.4357 & 49.6341 \\
\hline
\end{tabular}

TABLE 5: The directional statistic $\left(D_{\text {stat }}\right)$ values of different prediction models.

\begin{tabular}{|c|c|c|c|c|c|c|c|c|}
\hline Dataset & Horizon & MICEEMDAN -WOA-RVFL & RW & LSSVR & BPNN & ICEEMDAN -RW & ICEEMDAN -LSSVR & ICEEMDAN -BPNN \\
\hline \multirow{3}{*}{ WTI } & 1 & 0.9381 & 0.4815 & 0.5243 & 0.5191 & 0.4977 & 0.9190 & 0.9097 \\
\hline & 3 & 0.8576 & 0.4873 & 0.5087 & 0.5012 & 0.4907 & 0.8300 & 0.8449 \\
\hline & 6 & 0.7737 & 0.5116 & 0.4902 & 0.4948 & 0.5185 & 0.7714 & 0.7575 \\
\hline \multirow{3}{*}{ USD/EUR } & 1 & 0.9410 & 0.5019 & 0.4719 & 0.4897 & 0.5056 & 0.8998 & 0.9026 \\
\hline & 3 & 0.8502 & 0.4888 & 0.4897 & 0.4925 & 0.4916 & 0.8118 & 0.8024 \\
\hline & 6 & 0.7828 & 0.4991 & 0.5318 & 0.5253 & 0.5047 & 0.7023 & 0.7154 \\
\hline \multirow{3}{*}{ IP } & 1 & 0.9256 & 0.5579 & 0.5909 & 0.5785 & 0.5620 & 0.8636 & 0.8760 \\
\hline & 3 & 0.8802 & 0.6983 & 0.5455 & 0.4876 & 0.6529 & 0.8430 & 0.8058 \\
\hline & 6 & 0.8141 & 0.6198 & 0.5537 & 0.5661 & 0.6405 & 0.7355 & 0.7645 \\
\hline \multirow{3}{*}{ SSEC } & 1 & 0.9156 & 0.4944 & 0.5049 & 0.5098 & 0.4979 & 0.9024 & 0.9002 \\
\hline & 3 & 0.8396 & 0.5042 & 0.5021 & 0.5007 & 0.4965 & 0.8222 & 0.8145 \\
\hline & 6 & 0.7587 & 0.4833 & 0.5063 & 0.4965 & 0.4805 & 0.7448 & 0.7455 \\
\hline
\end{tabular}

performance. Of all the compared models, the proposed MICEEMDAN-WOA-RVFL obtains the highest $D_{\text {stat }}$ values and the lowest MAPE and RMSE values in all the time series datasets, showing that it is completely superior to the benchmark prediction models. Furthermore, for each prediction model, the MAPE and RMSE values increase while the $D_{\text {stat }}$ values decrease with the horizon. This demonstrates that it is easier to forecast time series with a short horizon than with a long one. It is worth noting that the proposed MICEEMDAN-WOA-RVFL still achieves the relatively good MAPE and RMSE with the increase of horizon among the compared models. For example, when the proposed model obtains $0.7737 D_{\text {stat }}$ with horizon 6 in the WTI dataset, it achieves the relatively low MAPE (0.0113) and RMSE (0.8146), indicating that the prediction values are very close to the real values although the proposed model misses direction about $22.63 \%$. In other words, the proposed MICEEMDANWOA-RVFL can still achieve satisfactory forecasting accuracy with a long horizon.

Furthermore, we can find that the multiple decomposition strategy does not improve the forecast for the RW model. One possible explanation is simply that RW infers that the past movement or trend of a time series cannot be used to predict its future movement, and thus, it cannot take advantage of the historical data and the diversity of multiple decomposition to make the future prediction. Therefore, when we aggregate the multiple RW model predictions of all the decomposed subseries, we just integrate several random predictions and, thus, cannot significantly improve the ensemble prediction results. In contrast, the LSSVR and
BPNN, as well as RVFL, can fully use all the historical data and the diversity of multiple decomposition to make the future prediction. Specifically, the multiple decomposition using different parameters generates many groups of different decomposed subseries, and the diversity of decomposition can successfully overcome the randomness of one single decomposition and further improve the prediction accuracy and stability of the developed forecasting approach.

In addition, the Diebold-Mariano (DM) test is utilized to evaluate whether the forecasting accuracy of the proposed MICEEMDAN-WOA-RVFL significantly outperforms those of the other compared models. Table 6 shows the statistics and $p$ values (in brackets).

On one hand, the DM statistical values between the ensemble prediction models and their corresponding single predictors are much lower than zero and the corresponding $p$ values are almost equal to zero with all the horizons except for the RW model, showing that the architecture of "decomposition and ensemble" contributes to greatly improving prediction accuracy and the combination of ICEEMDAN and AI predictors is more effective for economic and financial time series forecasting.

On the other hand, DM test results on the prediction of all the four time series datasets indicate that the MICEEMDAN-WOA-RVFL is significantly better than the single models and the other ensemble models with all the horizons, and the corresponding $p$ values are much lower than 0.01 in all the cases.

In summary, the DM test results demonstrate that the combination of multiple ICEEMDANs, RVFL networks, and 


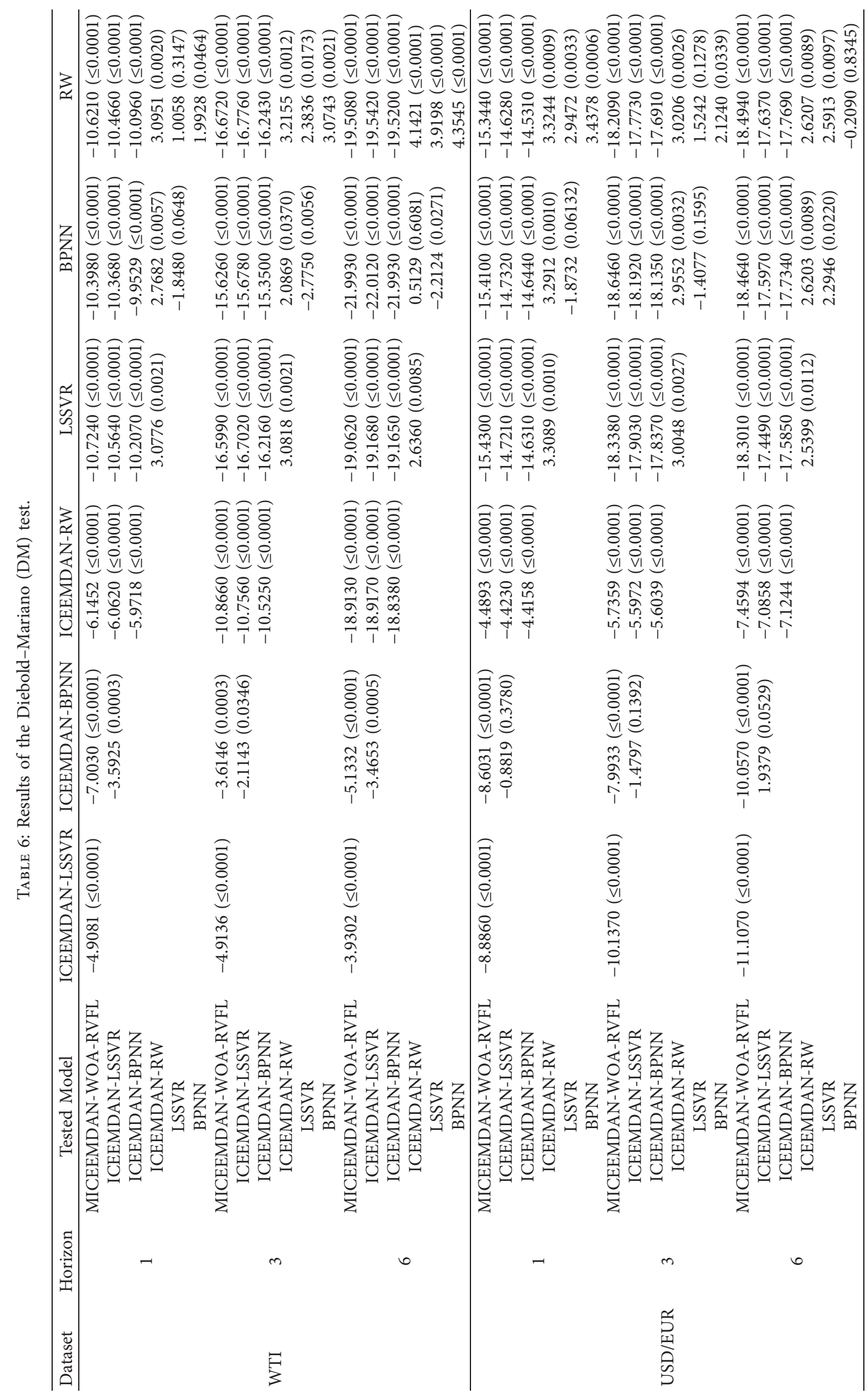




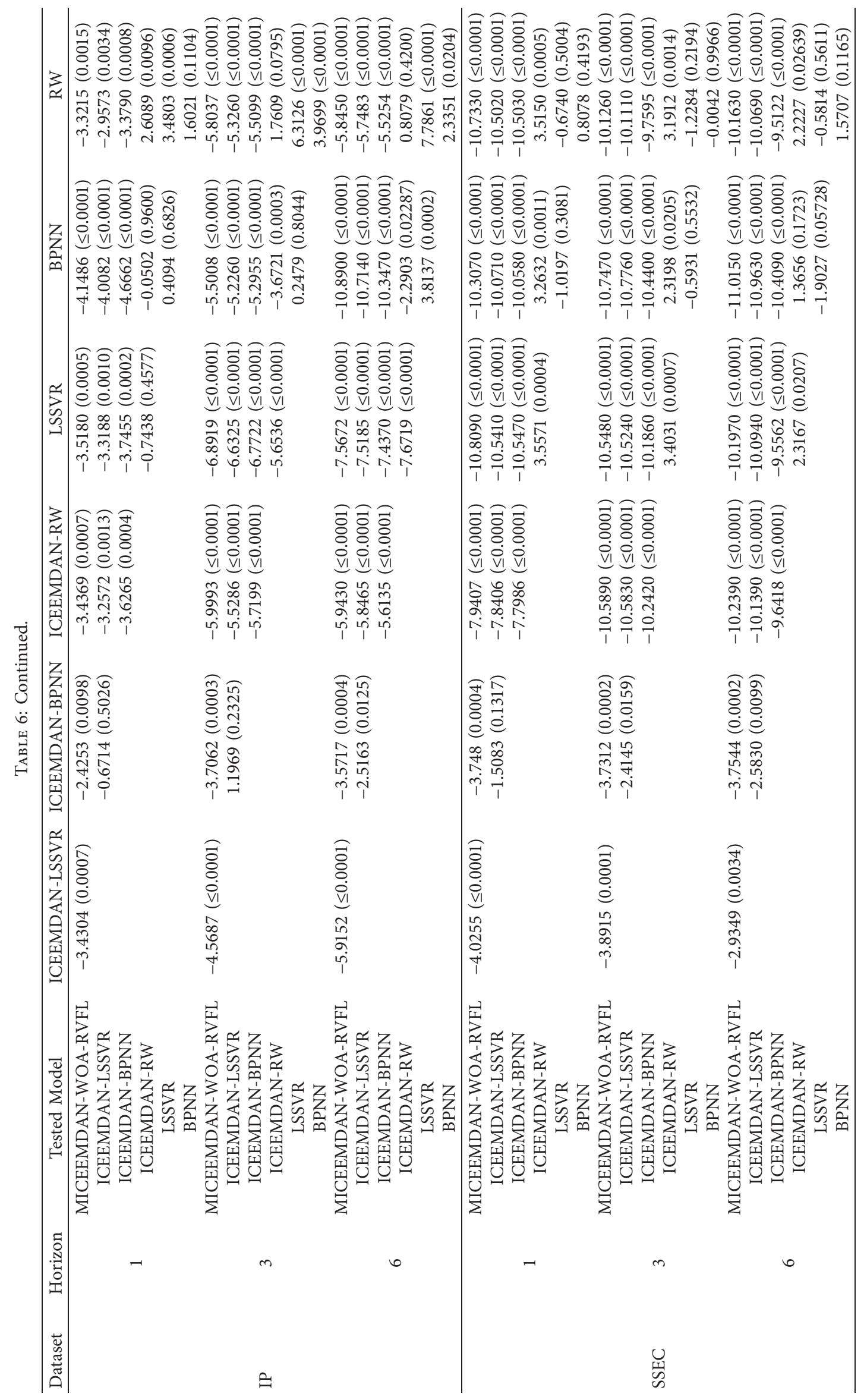


WOA optimization can significantly enhance the prediction accuracy of economic and financial time series forecasting.

\section{Discussion}

To better investigate the proposed MICEEMDAN-WOARVFL, we further discuss the developed prediction model, including the comparison of single decomposition and multiple decompositions, the optimization effectiveness of WOA, and the impact of ensemble size in this subsection.

5.1. Comparison of Single Decomposition and Multiple Decompositions. One of the main novelties of this study is the multiple decomposition strategy, which can successfully overcome the randomness of a single decomposition and improve the prediction accuracy and stability of the developed forecasting model. To evaluate the effectiveness of the multiple decomposition strategy, we compare the prediction results of MICEEMDAN-WOA-RVFL and ICEEMDAN-WOA-RVFL. The former ensembles the prediction results of $M(M=100)$ individual ICEEMDAN decompositions with random parameters, while the latter only employs one ICEEMDAN decomposition. We randomly choose 5 out of these 100 decompositions and execute ICEEMDAN-WOA-RVFL for time series forecasting. Tables 7-9 report the MAPE, RMSE, and $D_{\text {stat }}$ values of the MICEEMDAN-WOA-RVFL and the five ICEEMDANWOA-RVFL models using single decomposition and the corresponding mean values of these five models using single decomposition.

On one hand, compared with the prediction results of the five single decompositions and the mean prediction results, the proposed MICEEMDAN-WOA-RVFL achieves the lowest MAPE and the highest $D_{\text {stat }}$ values in all the 12 cases and the lowest RMSE values in 11 out of all the 12 cases, indicating that the multiple decomposition strategy can successfully overcome the randomness of single decomposition and improve the ensemble prediction accuracy.

On the other hand, we can find that the multiple decomposition strategy can greatly improve the stability of the prediction model. For example, the range of MAPE values of the five single decomposition models with horizon 6 in the IP time series dataset is from 0.0034 to 0.1114 , indicating that different single decomposition can produce relatively great difference in prediction results. When we employ the multiple decomposition strategy, we can overcome the randomness of single decomposition and, thus, enhance prediction stability.

In summary, the experimental results suggest that the multiple decomposition strategy and prediction ensemble can effectively enhance prediction accuracy and stability. The main reasons for the prediction improvement lie in three aspects: (1) the multiple decomposition can reduce the randomness of one single decomposition and simultaneously generate groups of differential subseries; (2) predictions using these groups of differential subseries can achieve diverse prediction results; and (3) the selection and ensemble of these diverse prediction results can ensure both accuracy and diversity and, thus, improve the final ensemble prediction.

5.2. The Optimization Effectiveness of WOA. When we use RVFL networks to construct predictors, a number of parameters need to be set in advance. In this study, WOA is introduced to search the optimal parameter values for RVFL predictors using its powerful optimization ability. To investigate the optimization effectiveness of WOA for parameter search, we compare the proposed MICEEMDAN-WOA-RVFL with MICEEMDAN-RVFL without WOA optimization. According to the literature [45], we fixed the number of hidden neurons $N_{h e}=100$, activation function Func = sigmoid, and random type Rand = Gaussian in MICEEMDAN-RVFL. The MAPE, RMSE, and $D_{\text {stat }}$ values are reported in Tables 10-12, respectively.

In all the four time series datasets, the prediction performance of the proposed MICEEMDAN-WOA-RVFL model is better than or equal to that of the MICEEMDANRVFL model without WOA optimization in all the 12 cases except for the RMSE value with horizon 1 in the SSEC dataset in terms of MAPE and RMSE, as listed in Tables 10 and 11. In addition, the MICEEMDAN-WOA-RVFL obtains the higher $D_{\text {stat }}$ values in 10 out of 12 cases, which can be seen in Table 12. The all results indicate that WOA can effectively search the optimal parameter settings for RVFL networks, further improving the overall prediction performance.

5.3. The Impact of Ensemble Size. The previous research has demonstrated that the ensemble strategy of using all individual prediction models is unlikely to work well and the selection of individual prediction models contributes to improving the ensemble prediction performance [57]. In this study, we sort all individual prediction models based on their past performance (RMSE values) and, then, select the top $N$ percent as the ensemble size to construct the ensemble prediction model. To further investigate the impact of ensemble size on ensemble prediction, we use different ensemble sizes (es $=10 \%, 20 \%, \ldots, 100 \%)$ to select the top $N$ percent of individual forecasting models to develop the ensemble prediction model and conduct the one-step-ahead forecasting experiment on the four time series datasets. The results are demonstrated in Figure 5.

We can see that the MICEEMDAN-WOA-RVFL obtains the best forecasting performance in the WTI, IP, and SSEC datasets when the ensemble size es is in the range of $20 \%-$ $40 \%$ and in the USD/EUR dataset when the ensemble size es 
TABLE 7: The mean absolute percent error (MAPE) values of single decomposition and multiple decompositions.

\begin{tabular}{|c|c|c|c|c|c|c|c|c|}
\hline Dataset & Horizon & $\begin{array}{l}\text { MICEEMDAN- } \\
\text { WOA-RVFL }\end{array}$ & $\begin{array}{l}\text { ICEEMDAN- } \\
\text { WOA-RVFL1 }\end{array}$ & $\begin{array}{l}\text { ICEEMDAN- } \\
\text { WOA-RVFL2 }\end{array}$ & $\begin{array}{l}\text { ICEEMDAN- } \\
\text { WOA-RVFL3 }\end{array}$ & $\begin{array}{l}\text { ICEEMDAN- } \\
\text { WOA-RVFL4 }\end{array}$ & $\begin{array}{l}\text { ICEEMDAN- } \\
\text { WOA-RVFL5 }\end{array}$ & Mean \\
\hline \multirow{3}{*}{ WTI } & 1 & 0.0036 & 0.0043 & 0.0045 & 0.0045 & 0.0041 & 0.0040 & 0.0043 \\
\hline & 3 & 0.0080 & 0.0088 & 0.0084 & 0.0084 & 0.0082 & 0.0083 & 0.0084 \\
\hline & 6 & 0.0113 & 0.0132 & 0.0115 & 0.0113 & 0.0118 & 0.0120 & 0.0112 \\
\hline \multirow{3}{*}{$\begin{array}{l}\text { USD/ } \\
\text { EUR }\end{array}$} & 1 & 0.0006 & 0.0011 & 0.0010 & 0.0008 & 0.0010 & 0.0008 & 0.0009 \\
\hline & 3 & 0.0015 & 0.0021 & 0.0021 & 0.0015 & 0.0017 & 0.0015 & 0.0018 \\
\hline & 6 & 0.0022 & 0.0033 & 0.0022 & 0.0023 & 0.0023 & 0.0024 & 0.0025 \\
\hline \multirow{3}{*}{ IP } & 1 & 0.0012 & 0.0016 & 0.0016 & 0.0017 & 0.0017 & 0.0015 & 0.0016 \\
\hline & 3 & 0.0023 & 0.0024 & 0.0026 & 0.0030 & 0.0028 & 0.0024 & 0.0026 \\
\hline & 6 & 0.0032 & 0.0036 & 0.0034 & 0.1114 & 0.0036 & 0.0041 & 0.0252 \\
\hline \multirow{3}{*}{ SSEC } & 1 & 0.0020 & 0.0024 & 0.0023 & 0.0026 & 0.0023 & 0.0023 & 0.0024 \\
\hline & 3 & 0.0044 & 0.0045 & 0.0046 & 0.0052 & 0.0045 & 0.0047 & 0.0047 \\
\hline & 6 & 0.0065 & 0.0067 & 0.0070 & 0.0085 & 0.0068 & 0.0075 & 0.0073 \\
\hline
\end{tabular}

TABLE 8: The root mean squared error (RMSE) values of single decomposition and multiple decompositions.

\begin{tabular}{|c|c|c|c|c|c|c|c|c|}
\hline Dataset & Horizon & $\begin{array}{l}\text { MICEEMDAN- } \\
\text { WOA-RVFL }\end{array}$ & $\begin{array}{l}\text { ICEEMDAN- } \\
\text { WOA-RVFL1 }\end{array}$ & $\begin{array}{l}\text { ICEEMDAN- } \\
\text { WOA-RVFL2 }\end{array}$ & $\begin{array}{l}\text { ICEEMDAN- } \\
\text { WOA-RVFL3 }\end{array}$ & $\begin{array}{l}\text { ICEEMDAN- } \\
\text { WOA-RVFL4 }\end{array}$ & $\begin{array}{l}\text { ICEEMDAN- } \\
\text { WOA-RVFL5 }\end{array}$ & Mean \\
\hline \multirow{3}{*}{ WTI } & 1 & 0.2715 & 0.3316 & 0.3376 & 0.3352 & 0.3191 & 0.3148 & 0.3277 \\
\hline & 3 & 0.5953 & 0.6557 & 0.6214 & 0.6200 & 0.6058 & 0.6133 & 0.6232 \\
\hline & 6 & 0.8146 & 0.9490 & 0.8239 & 0.8199 & 0.8508 & 0.8597 & 0.8607 \\
\hline \multirow{3}{*}{$\begin{array}{l}\text { USD/ } \\
\text { EUR }\end{array}$} & 1 & 0.0009 & 0.0017 & 0.0017 & 0.0012 & 0.0015 & 0.0012 & 0.0015 \\
\hline & 3 & 0.0022 & 0.0031 & 0.0034 & 0.0023 & 0.0025 & 0.0023 & 0.0027 \\
\hline & 6 & 0.0033 & 0.0048 & 0.0033 & 0.0035 & 0.0035 & 0.0036 & 0.0037 \\
\hline \multirow{3}{*}{ IP } & 1 & 0.2114 & 0.2894 & 0.3080 & 0.3656 & 0.3220 & 0.2589 & 0.3088 \\
\hline & 3 & 0.3875 & 0.4098 & 0.4507 & 0.4809 & 0.4776 & 0.4027 & 0.4443 \\
\hline & 6 & 0.5340 & 0.5502 & 0.5563 & 1.0081 & 0.5697 & 0.6466 & 0.6661 \\
\hline \multirow{3}{*}{ SSEC } & 1 & 10.8115 & 11.5515 & 13.0728 & 16.7301 & 12.2465 & 14.3671 & 13.5936 \\
\hline & 3 & 22.2983 & 22.0603 & 22.8140 & 33.9937 & 23.9755 & 25.8004 & 25.7288 \\
\hline & 6 & 35.7201 & 37.0255 & 41.9033 & 55.5066 & 38.6140 & 48.2131 & 44.2525 \\
\hline
\end{tabular}

TABLE 9: The directional statistic $\left(D_{\text {stat }}\right)$ values of single decomposition and multiple decompositions.

\begin{tabular}{|c|c|c|c|c|c|c|c|c|}
\hline Dataset & Horizon & $\begin{array}{l}\text { MICEEMDAN- } \\
\text { WOA-RVFL }\end{array}$ & $\begin{array}{l}\text { ICEEMDAN- } \\
\text { WOA-RVFL1 }\end{array}$ & $\begin{array}{l}\text { ICEEMDAN- } \\
\text { WOA-RVFL2 }\end{array}$ & $\begin{array}{l}\text { ICEEMDAN- } \\
\text { WOA-RVFL3 }\end{array}$ & $\begin{array}{l}\text { ICEEMDAN- } \\
\text { WOA-RVFL4 }\end{array}$ & $\begin{array}{l}\text { ICEEMDAN- } \\
\text { WOA-RVFL5 }\end{array}$ & Mean \\
\hline \multirow{3}{*}{ WTI } & 1 & 0.9381 & 0.9201 & 0.9161 & 0.9184 & 0.9323 & 0.9271 & 0.9228 \\
\hline & 3 & 0.8576 & 0.8414 & 0.8548 & 0.8385 & 0.8553 & 0.8513 & 0.8483 \\
\hline & 6 & 0.7737 & 0.7419 & 0.7703 & 0.7668 & 0.7627 & 0.7616 & 0.7607 \\
\hline \multirow{3}{*}{$\begin{array}{l}\text { USD/ } \\
\text { EUR }\end{array}$} & 1 & 0.941 & 0.9008 & 0.9270 & 0.9204 & 0.9073 & 0.9157 & 0.9142 \\
\hline & 3 & 0.8502 & 0.8052 & 0.8418 & 0.8399 & 0.8296 & 0.8427 & 0.8318 \\
\hline & 6 & 0.7828 & 0.6826 & 0.7809 & 0.7819 & 0.7772 & 0.7706 & 0.7586 \\
\hline \multirow{3}{*}{ IP } & 1 & 0.9256 & 0.8967 & 0.8926 & 0.9132 & 0.8967 & 0.9174 & 0.9033 \\
\hline & 3 & 0.8802 & 0.8760 & 0.8430 & 0.8223 & 0.8471 & 0.8802 & 0.8537 \\
\hline & 6 & 0.8141 & 0.8017 & 0.8017 & 0.7066 & 0.7851 & 0.8017 & 0.7794 \\
\hline \multirow{3}{*}{ SSEC } & 1 & 0.9156 & 0.9052 & 0.9052 & 0.9052 & 0.9087 & 0.9135 & 0.9076 \\
\hline & 3 & 0.8396 & 0.8222 & 0.8292 & 0.8208 & 0.8368 & 0.8264 & 0.8271 \\
\hline & 6 & 0.7587 & 0.7462 & 0.7441 & 0.7134 & 0.7594 & 0.7448 & 0.7416 \\
\hline
\end{tabular}

TABLE 10: The mean absolute percent error (MAPE) values with and without WOA optimization.

\begin{tabular}{lccc}
\hline Dataset & Horizon & MICEEMDAN-WOA-RVFL & MICEEMDAN-RVFL \\
\hline \multirow{2}{*}{ WTI } & 1 & $\mathbf{0 . 0 0 3 6}$ & 0.0037 \\
& 3 & $\mathbf{0 . 0 0 8 0}$ & 0.0082 \\
\hline \multirow{2}{*}{ USD/EUR } & 6 & $\mathbf{0 . 0 1 1 3}$ & 0.0118 \\
& 1 & $\mathbf{0 . 0 0 0 6}$ & 0.0006 \\
\hline \multirow{3}{*}{ IP } & 3 & $\mathbf{0 . 0 0 1 5}$ & 0.0015 \\
& 6 & $\mathbf{0 . 0 0 2 2}$ & 0.0023 \\
\hline & 1 & $\mathbf{0 . 0 0 1 2}$ & 0.0013 \\
SSEC & 3 & $\mathbf{0 . 0 0 2 3}$ & 0.0023 \\
& 6 & $\mathbf{0 . 0 0 3 2}$ & 0.0036 \\
\hline
\end{tabular}


TABLE 11: The root mean squared error (RMSE) values with and without WOA optimization.

\begin{tabular}{lccc}
\hline Dataset & Horizon & MICEEMDAN-WOA-RVFL & MICEEMDAN-RVFL \\
\hline \multirow{2}{*}{ WTI } & 1 & $\mathbf{0 . 2 7 1 5}$ & 0.2824 \\
& 3 & $\mathbf{0 . 5 9 5 3}$ & 0.6079 \\
\hline \multirow{2}{*}{ USD/EUR } & 6 & $\mathbf{0 . 8 1 4 6}$ & 0.8485 \\
& 1 & $\mathbf{0 . 0 0 0 9}$ & 0.0010 \\
\hline \multirow{2}{*}{ IP } & 3 & $\mathbf{0 . 0 0 2 2}$ & 0.0022 \\
& 6 & $\mathbf{0 . 0 0 3 3}$ & 0.0034 \\
\hline \multirow{3}{*}{ SSEC } & 1 & $\mathbf{0 . 2 1 1 4}$ & 0.2362 \\
& 3 & $\mathbf{0 . 3 8 7 5}$ & 0.4033 \\
& 6 & $\mathbf{0 . 5 3 4 0}$ & 0.5531 \\
\hline
\end{tabular}

TABLE 12: The directional statistic $\left(D_{\text {stat }}\right)$ values with and without WOA optimization.

\begin{tabular}{lccc}
\hline Dataset & Horizon & MICEEMDAN-WOA-RVFL & MICEEMDAN-RVFL \\
\hline \multirow{2}{*}{ WTI } & 1 & $\mathbf{0 . 9 3 8 1}$ & 0.9358 \\
& 3 & $\mathbf{0 . 8 5 7 6}$ & 0.8565 \\
\hline \multirow{2}{*}{ USD/EUR } & 6 & $\mathbf{0 . 7 7 3 7}$ & 0.7656 \\
& 1 & $\mathbf{0 . 9 4 1}$ & 0.9317 \\
\hline \multirow{3}{*}{ IP } & 3 & $\mathbf{0 . 8 5 0 2}$ & 0.8455 \\
& 6 & $\mathbf{0 . 7 8 2 8}$ & 0.7762 \\
\hline \multirow{2}{*}{ SSEC } & 1 & $\mathbf{0 . 9 2 5 6}$ & 0.9174 \\
& 3 & $\mathbf{0 . 8 8 0 2}$ & 0.8430 \\
& 6 & $\mathbf{0 . 8 1 4 1}$ & 0.7893 \\
\hline
\end{tabular}

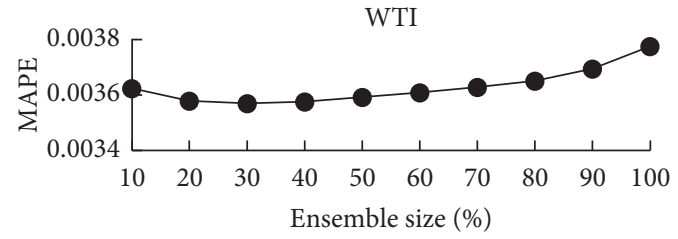

(a)

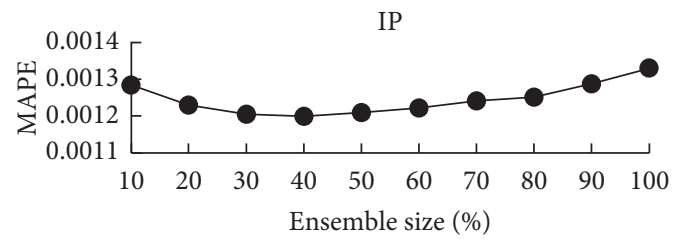

(c)

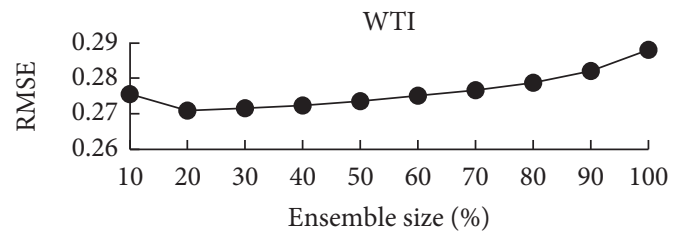

(e)

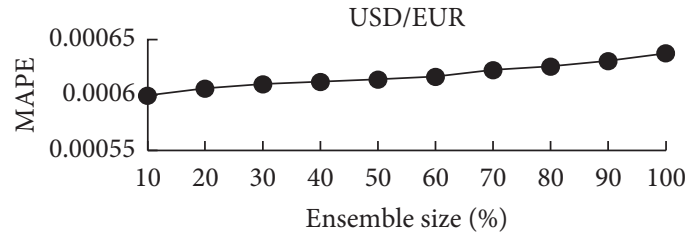

(b)

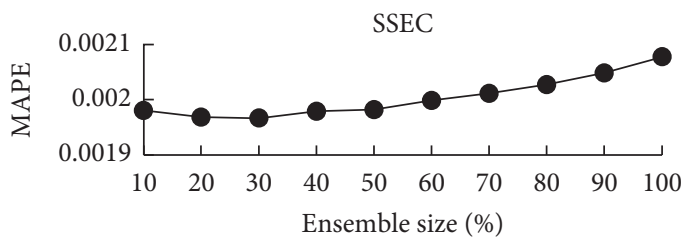

(d)

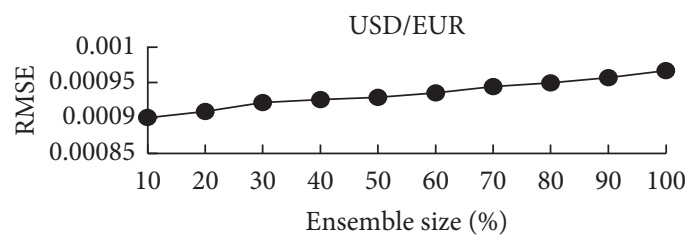

(f)

FIGURE 5: Continued. 


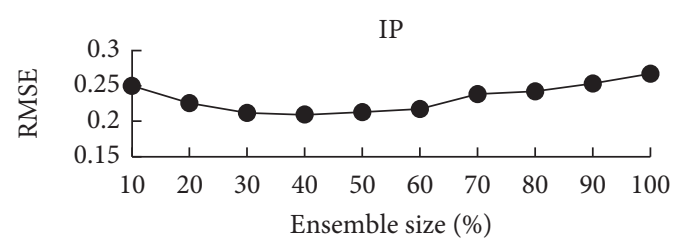

(g)

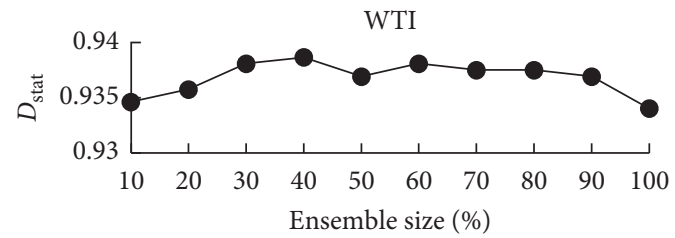

(i)

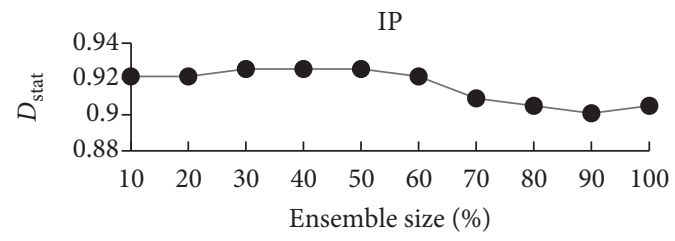

(k)

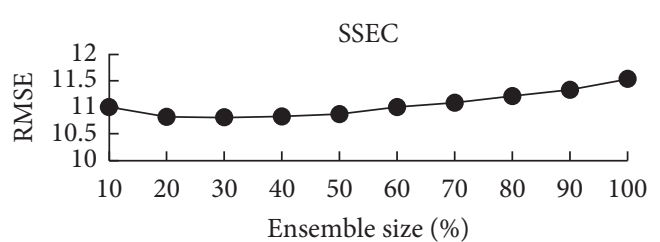

(h)

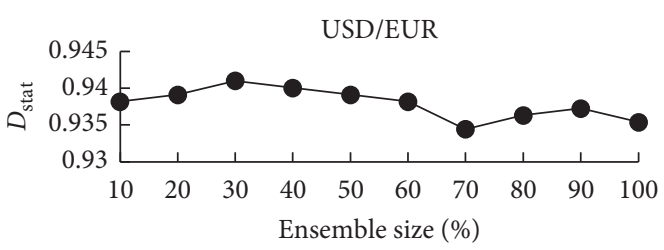

(j)

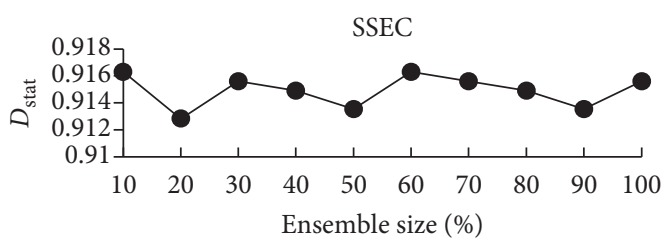

(1)

Figure 5: The impact of ensemble size with one-step-ahead forecasting.

is in the range of $10 \%-40 \%$ in terms of MAPE, RMSE, and $D_{\text {stat }}$. When the es is greater than $40 \%$, the MAPE and RMSE values continue to worsen and become the worst when the ensemble size grows to 100 . The experimental results indicate that the ensemble size has an overall significant impact on ensemble prediction, and an ideal range of ensemble size is about $20 \%$ to $40 \%$.

\section{Conclusions}

To better forecast economic and financial time series, we propose a novel multidecomposition and self-optimizing ensemble prediction model MICEEMDAN-WOA-RVFL combining multiple ICEEMDANs, WOA, and RVFL networks. The MICEEMDAN-WOA-RVFL first uses ICEEMDAN to multiply separate original economic and financial time series into groups of subseries many times, and then, RVFL networks are used to individually forecast the decomposed subseries in each decomposition. Simultaneously, WOA is introduced to optimize RVFL networks to further improve the prediction accuracy. Thirdly, the predictions of subseries in each decomposition are integrated into the forecasting results of each decomposition using addition. Finally, the prediction results of each decomposition are selected based on RMSE values and are combined as the final prediction results.

As far as we know, it is the first time that WOA is employed for the optimal parameter search for RVFL networks and the multiple decomposition strategy is introduced in time series forecasting. The empirical results indicate that
(1) the proposed MICEEMDAN-WOA-RVFL significantly improves prediction accuracy in various economic and financial time series forecasting; (2) WOA can effectively search optimal parameters for RVFL networks and improve prediction performance of economic and financial time series forecasting; and (3) the multiple decomposition strategy can successfully overcome the randomness of a single decomposition and enhance the prediction accuracy and stability of the developed prediction model.

We will extend our study in two aspects in the future: (1) applying the MICEEMDAN-WOA-RVFL to forecast more economic and financial time series and (2) improving the selection and ensemble method of individual forecasting models to further enhance the prediction performance.

\section{Data Availability}

The data used to support the findings of this study are included within the article.

\section{Conflicts of Interest}

The authors declare that they have no conflicts of interest.

\section{Acknowledgments}

This work was supported by the Fundamental Research Funds for the Central Universities (Grant no. JBK2003001), the Ministry of Education of Humanities and Social Science Project (Grant nos. 19YJAZH047 and 16XJAZH002), and 
the Scientific Research Fund of Sichuan Provincial Education Department (Grant no. 17ZB0433).

\section{References}

[1] Y. Hui, W.-K. Wong, Z. Bai, and Z.-Z. Zhu, "A new nonlinearity test to circumvent the limitation of Volterra expansion with application," Journal of the Korean Statistical Society, vol. 46, no. 3, pp. 365-374, 2017.

[2] R. Adhikari and R. K. Agrawal, "A combination of artificial neural network and random walk models for financial time series forecasting," Neural Computing and Applications, vol. 24, no. 6, pp. 1441-1449, 2014.

[3] A. Lanza, M. Manera, and M. Giovannini, "Modeling and forecasting cointegrated relationships among heavy oil and product prices," Energy Economics, vol. 27, no. 6, pp. 831-848, 2005.

[4] M. R. Hassan and B. Nath, "Stock market forecasting using hidden Markov model: a new approach," in Proceedings of the 5th International Conference on Intelligent Systems Design and Applications (ISDA'05), pp. 192-196, IEEE, Warsaw, Poland, September 2005.

[5] L. Kilian and M. P. Taylor, "Why is it so difficult to beat the random walk forecast of exchange rates?" Journal of International Economics, vol. 60, no. 1, pp. 85-107, 2003.

[6] M. Rout, B. Majhi, R. Majhi, and G. Panda, "Forecasting of currency exchange rates using an adaptive ARMA model with differential evolution based training," Journal of King Saud University-Computer and Information Sciences, vol. 26, no. 1, pp. 7-18, 2014.

[7] P. Mondal, L. Shit, and S. Goswami, "Study of effectiveness of time series modeling (ARIMA) in forecasting stock prices," International Journal of Computer Science, Engineering and Applications, vol. 4, no. 2, pp. 13-29, 2014.

[8] A. A. Drakos, G. P. Kouretas, and L. P. Zarangas, "Forecasting financial volatility of the Athens stock exchange daily returns: an application of the asymmetric normal mixture GARCH model," International Journal of Finance \& Economics, vol. 15, pp. 331-350, 2010.

[9] D. Alberg, H. Shalit, and R. Yosef, "Estimating stock market volatility using asymmetric GARCH models," Applied Financial Economics, vol. 18, no. 15, pp. 1201-1208, 2008.

[10] R. P. Pradhan and R. Kumar, "Forecasting exchange rate in India: an application of artificial neural network model," Journal of Mathematics Research, vol. 2, pp. 111-117, 2010.

[11] S. T. A. Niaki and S. Hoseinzade, "Forecasting S\&P 500 index using artificial neural networks and design of experiments," Journal of Industrial Engineering International, vol. 9, pp. 1-9, 2013.

[12] S. P. Das and S. Padhy, "A novel hybrid model using teachinglearning-based optimization and a support vector machine for commodity futures index forecasting," International Journal of Machine Learning and Cybernetics, vol. 9, no. 1, pp. 97-111, 2018.

[13] M. K. Okasha, "Using support vector machines in financial time series forecasting," International Journal of Statistics and Applications, vol. 4, pp. 28-39, 2014.

[14] X. Li, H. Xie, R. Wang et al., "Empirical analysis: stock market prediction via extreme learning machine," Neural Computing and Applications, vol. 27, no. 1, pp. 67-78, 2016.
[15] T. Moudiki, F. Planchet, and A. Cousin, "Multiple time series forecasting using quasi-randomized functional link neural networks," Risks, vol. 6, no. 1, pp. 22-42, 2018.

[16] Y. Baek and H. Y. Kim, "ModAugNet: a new forecasting framework for stock market index value with an overfitting prevention LSTM module and a prediction LSTM module," Expert Systems with Applications, vol. 113, pp. 457-480, 2018.

[17] C. N. Babu and B. E. Reddy, "A moving-average filter based hybrid ARIMA-ANN model for forecasting time series data," Applied Soft Computing, vol. 23, pp. 27-38, 2014.

[18] M. Kumar and M. Thenmozhi, "Forecasting stock index returns using ARIMA-SVM, ARIMA-ANN, and ARIMArandom forest hybrid models," International Journal of Banking, Accounting and Finance, vol. 5, no. 3, pp. 284-308, 2014.

[19] C.-M. Hsu, "A hybrid procedure with feature selection for resolving stock/futures price forecasting problems," Neural Computing and Applications, vol. 22, no. 3-4, pp. 651-671, 2013.

[20] S. Lahmiri, "A variational mode decompoisition approach for analysis and forecasting of economic and financial time series," Expert Systems with Applications, vol. 55, pp. 268-273, 2016.

[21] L.-J. Kao, C.-C. Chiu, C.-J. Lu, and C.-H. Chang, "A hybrid approach by integrating wavelet-based feature extraction with MARS and SVR for stock index forecasting," Decision Support Systems, vol. 54, no. 3, pp. 1228-1244, 2013.

[22] T. Li, Y. Zhou, X. Li, J. Wu, and T. He, "Forecasting daily crude oil prices using improved CEEMDAN and ridge regression-based predictors," Energies, vol. 12, no. 19, pp. 3603-3628, 2019.

[23] A. Bagheri, H. Mohammadi Peyhani, and M. Akbari, "Financial forecasting using ANFIS networks with quantumbehaved particle swarm optimization," Expert Systems with Applications, vol. 41, no. 14, pp. 6235-6250, 2014.

[24] J. Wang, R. Hou, C. Wang, and L. Shen, "Improved v-Support vector regression model based on variable selection and brain storm optimization for stock price forecasting," Applied Soft Computing, vol. 49, pp. 164-178, 2016.

[25] G. Claeskens, J. R. Magnus, A. L. Vasnev, and W. Wang, "The forecast combination puzzle: a simple theoretical explanation," International Journal of Forecasting, vol. 32, no. 3, pp. 754-762, 2016.

[26] S. G. Hall and J. Mitchell, "Combining density forecasts," International Journal of Forecasting, vol. 23, no. 1, pp. 1-13, 2007.

[27] N. E. Huang, Z. Shen, S. R. Long et al., "The empirical mode decomposition and the Hilbert spectrum for nonlinear and non-stationary time series analysis," Proceedings of the Royal Society of London. Series A: Mathematical, Physical and Engineering Sciences, vol. 454, no. 1971, pp. 903-995, 1998.

[28] Z. Wu and N. E. Huang, "Ensemble empirical mode decomposition: a noise-assisted data analysis method," Advances in Adaptive Data Analysis, vol. 1, no. 1, pp. 1-41, 2009.

[29] M. E. Torres, M. A. Colominas, G. Schlotthauer, and P. Flandrin, "A complete ensemble empirical mode decomposition with adaptive noise," in Proceedings of the 2011 IEEE International Conference on Acoustics, Speech and Signal Processing (ICASSP), pp. 4144-4147, IEEE, Prague, Czech Republic, May 2011. 
[30] J. Wu, T. Zhou, and T. Li, "Detecting epileptic seizures in EEG signals with complementary ensemble empirical mode decomposition and extreme gradient boosting," Entropy, vol. 22, no. 2, pp. 140-165, 2020.

[31] T. Li, Z. Hu, Y. Jia, J. Wu, and Y. Zhou, "Forecasting crude oil prices using ensemble empirical mode decomposition and sparse Bayesian learning," Energies, vol. 11, no. 7, pp. 1882-1905, 2018.

[32] T. Li, M. Zhou, C. Guo et al., "Forecasting crude oil price using EEMD and RVM with adaptive PSO-based kernels," Energies, vol. 9, no. 12, p. 1014, 2016.

[33] M. A. Colominas, G. Schlotthauer, and M. E. Torres, "Improved complete ensemble EMD: a suitable tool for biomedical signal processing," Biomedical Signal Processing and Control, vol. 14, pp. 19-29, 2014.

[34] J. Wu, F. Miu, and T. Li, "Daily crude oil price forecasting based on improved CEEMDAN, SCA, and RVFL: a case study in WTI oil market," Energies, vol. 13, no. 7, pp. 1852-1872, 2020.

[35] T. Li, Z. Qian, and T. He, "Short-term load forecasting with improved CEEMDAN and GWO-based multiple kernel ELM," Complexity, vol. 2020, Article ID 1209547, 20 pages, 2020.

[36] W. Yang, J. Wang, T. Niu, and P. Du, "A hybrid forecasting system based on a dual decomposition strategy and multiobjective optimization for electricity price forecasting," Applied Energy, vol. 235, pp. 1205-1225, 2019.

[37] W. Deng, J. Xu, Y. Song, and H. Zhao, "An effective improved co-evolution ant colony optimization algorithm with multistrategies and its application," International Journal of BioInspired Computation, vol. 16, pp. 1-10, 2020.

[38] S. Mirjalili and A. Lewis, "The whale optimization algorithm," Advances in Engineering Software, vol. 95, pp. 51-67, 2016.

[39] I. Aljarah, H. Faris, and S. Mirjalili, "Optimizing connection weights in neural networks using the whale optimization algorithm," Soft Computing, vol. 22, no. 1, pp. 1-15, 2018.

[40] J. Wang, P. Du, T. Niu, and W. Yang, "A novel hybrid system based on a new proposed algorithm-Multi-Objective Whale Optimization Algorithm for wind speed forecasting," Applied Energy, vol. 208, pp. 344-360, 2017.

[41] Z. Alameer, M. A. Elaziz, A. A. Ewees, H. Ye, and Z. Jianhua, "Forecasting gold price fluctuations using improved multilayer perceptron neural network and whale optimization algorithm," Resources Policy, vol. 61, pp. 250-260, 2019.

[42] Y.-H. Pao, G.-H. Park, and D. J. Sobajic, "Learning and generalization characteristics of the random vector functional-link net," Neurocomputing, vol. 6, no. 2, pp. 163-180, 1994.

[43] L. Zhang and P. N. Suganthan, "A comprehensive evaluation of random vector functional link networks," Information Sciences, vol. 367-368, pp. 1094-1105, 2016.

[44] Y. Ren, P. N. Suganthan, N. Srikanth, and G. Amaratunga, "Random vector functional link network for short-term electricity load demand forecasting," Information Sciences, vol. 367-368, pp. 1078-1093, 2016.

[45] L. Tang, Y. Wu, and L. Yu, "A non-iterative decompositionensemble learning paradigm using RVFL network for crude oil price forecasting," Applied Soft Computing, vol. 70, pp. 1097-1108, 2018.

[46] T. Li, J. Shi, X. Li, J. Wu, and F. Pan, "Image encryption based on pixel-level diffusion with dynamic filtering and DNA-level permutation with 3D Latin cubes," Entropy, vol. 21, no. 3, pp. 319-340, 2019.
[47] J. Wu, J. Shi, and T. Li, “A novel image encryption approach based on a hyperchaotic system, pixel-level filtering with variable kernels, and DNA-level diffusion," Entropy, vol. 22, pp. 5-24, 2020.

[48] T. Li, M. Yang, J. Wu, and X. Jing, "A novel image encryption algorithm based on a fractional-order hyperchaotic system and DNA computing," Complexity, vol. 2017, Article ID 9010251, 13 pages, 2017.

[49] H. Zhao, J. Zheng, W. Deng, and Y. Song, "Semi-supervised broad learning system based on manifold regularization and broad network," IEEE Transactions on Circuits and Systems I: Regular Papers, vol. 67, no. 3, pp. 983-994, 2020.

[50] S. Sun, S. Wang, and Y. Wei, "A new multiscale decomposition ensemble approach for forecasting exchange rates," Economic Modelling, vol. 81, pp. 49-58, 2019.

[51] T. Li and M. Zhou, "ECG classification using wavelet packet entropy and random forests," Entropy, vol. 18, no. 8, p. 285, 2016.

[52] H. Zhao, H. Liu, J. Xu, and W. Deng, "Performance prediction using high-order differential mathematical morphology gradient spectrum entropy and extreme learning machine," IEEE Transactions on Instrumentation and Measurement, vol. 69, pp. 4165-4172, 2019.

[53] W. Deng, H. Liu, J. Xu, H. Zhao, and Y. Song, "An improved quantum-inspired differential evolution algorithm for deep belief network," IEEE Transactions on Instrumentation and Measurement, vol. 69, no. 10, pp. 7319-7327, 2020.

[54] H. Liu, Z. Duan, F.-Z. Han, and Y.-F. Li, "Big multi-step wind speed forecasting model based on secondary decomposition, ensemble method and error correction algorithm," Energy Conversion and Management, vol. 156, pp. 525-541, 2018.

[55] Fred Website. https://fred.stlouisfed.org/.

[56] NetEase Website. http://quotes.money.163.com/service/ chddata.html . code $=0000001$ \&start $=19901219$.

[57] M. Aiolfi and A. Timmermann, "Persistence in forecasting performance and conditional combination strategies," Journal of Econometrics, vol. 135, no. 1-2, pp. 31-53, 2006. 